\title{
Von Daten und Datensicherheit
}

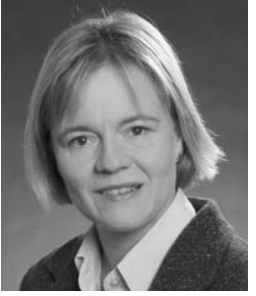

E. Hummers-Pradier

Bibliografie

DOI 10.1055/s-0028-1100377

Z Allg Med 2008; 84: 465-466

(c) Georg Thieme Verlag KG

Stuttgart · New York

ISSN 1433-6251

Korrespondenzadresse

Prof. Dr. med. E. Hummers-

\section{Pradier}

Institut für Allgemeinmedizin

Medizinische Hochschule

Hannover

Carl-Neuberg-Straße 1

30625 Hannover

Hummers-pradier.eva@

mh-hannover.de
Negative Schlagzeilen in den letzten 3 Monaten: Weitergabe von 200000 Datensätzen chronisch kranker Versicherter der Deutschen Angestellten Krankenkasse an die Privatfirma Healthways (19.08.2008). Daten von 17 Millionen Kunden der Telekom wurden entwendet (6.10.2008).

Positive Schlagzeile (vielleicht in einigen Monaten): Einführung der elektronischen Gesundheitskarte führt zu mehr Sicherheit und Transparenz im Gesundheitswesen.

So optimistisch sieht das zumindest die Gesellschaft für Teleanwendungen im Gesundheitswesen (Dematik), die auf ihrer Homepage mit den Worten wirbt: „Die elektronische Gesundheitskarte ist die Voraussetzung für eine grundlegende Modernisierung des deutschen Gesundheitswesens. Sie ist der Schlüssel zu einer Vielzahl von neuen Funktionen und wird in Verbindung mit einer Telematikinfrastruktur für die Datenkommunikation die Qualität der medizinischen Versorgung erhöhen und das Recht auf Eigenverantwortung der Patienten stärken“ (www.Gematik.de).

Derzeit sind die Vorbereitungen für das BasisRollout der elektronischen Gesundheitskarte in Nordrhein ins Stocken geraten, weil bisher nicht alle mobilen Lesegeräte von der Gematik zugelassen wurden. Nun wird damit gerechnet, dass bis Ende November alle auf dem Markt befindlichen stationären wie mobilen Geräte zertifiziert sein werden. Allerdings steht bisher auch die Festsetzung der Pauschale aus, die niedergelassene Ärzte für die Anschaffung und Installation der Lesegeräte erhalten sollen. Mit dem Start der elektronischen Gesundheitskarte außerhalb von Modellprojekten ist daher kaum vor Beginn des nächsten Jahres zu rechnen. Ist das nun eine gute oder eine schlechte Nachricht?

Einige ältere Patienten, die im Rahmen eines Forschungsprojekts des Hannoveraner Instituts für Allgemeinmedizin von uns besucht wurden, waren sehr besorgt, ob wir wohl zuverlässig mit ihren persönlichen Daten umgehen. So bestanden sie darauf, dass bei der Aufzeichnung eines Interviews ihre Namen nicht mit registriert wurden, und überprüften dies auch, in dem sie sich den Anfang der Aufnahme vorspielen ließen. Polaroid-Fotos wurden uns nicht zugestanden, aus Sorge, wir würden damit nicht korrekt umgehen. Dabei brachten diese Patienten unserer Studienärztin durchaus Wohlwollen entgegen. Sie äußerten jedoch starke Bedenken, ob in Zukunft mit ihren Daten auf einer elektronischen Patientenkarte adäquat umgegangen wird, und in wessen Hände ihre persönlichen Daten denn wohl gelangen könnten.
Auch unter Ärzten mangelt es nicht an kritischen Stimmen: „Wer kann jetzt noch von „Sicherheit der gespeicherten Daten“ sprechen, ohne sich lächerlich zu machen?" so der Landesverband Nordrhein des NAV-Virchow-Bundes, Verband der niedergelassenen Ärzte Deutschlands (6.10.2008) Ein Blick ins Ausland zeigt, dass in den USA sind „electronic personal health records“ bereits recht weit verbreitet sind. Health Care Organizations wie Kaiser Permanente bieten ihren Versicherten das Speichern persönlicher Gesundheitsdaten an, die dann für sämtliche Ärzte des Systems jederzeit und an jedem Ort abrufbar sind. Microsoft unterhält mit healthvault.com seit Oktober 2007 ein allgemein zugängliches Portal, dass seinen Mitgliedern die Möglichkeit bietet, ihre persönlichen Gesundheitsdaten so zu hinterlegen, dass sie für viele Gesundheitsanbieter abrufbar sind. Google Health ist mit einem ähnlichen Ansatz im Mai dieses Jahres gefolgt. Hier können die Nutzer ihren Ärzten sogar erlauben, neuerhobene Daten direkt in das System einzugeben und können sich somit zeitaufwendige Eingaben am heimischen PC ersparen. Dort haben sie auch die Möglichkeit, ihre Dokumente zu sichten und zu organisieren und weiteren Ärzten zugänglich zu machen. Allerdings sind bisher nicht mehr als 20 medizinische Anbieter in den USA Partner von Google-Health. Bezüglich der Datensicherheit erheben Kritiker starke Bedenken. Sie fürchten, dass interessierte Parteien wie Versicherungen und Arbeitgeber Wege finden werden, an die sensiblen Gesundheitsdaten zu gelangen. Die Kritiker warnen zudem davor, dass Suchmaschinenbetreiber wie Google nicht dem Gesundheitsdatenschutz unterliegen und somit im Falle einer Schädigung kaum Regressmöglichkeiten bestehen werden. Dennoch: Der Trend zum Nutzen dieser Portale seitens der Verbraucher hält an. In den Niederlanden wird eine elektronische Akte ohne E-Card geplant. In einem ersten Schritt wird eine elektronische Medikationsakte eingeführt, folgen soll eine Vertretungsakte für die Hausärzte, in der alle relevanten medizinischen Basisdaten der Patienten enthalten sein sollen. Ärzte im Notdienst haben so die Möglichkeit, sich schnell ein umfassendes Bild über den Patienten zu machen. Die Verwaltung wird über zentrale Bürgerservicenummer erfolgen, weder wird eine Chipkarte wie bei uns, noch eine Geheimnummer wie bei den Online- Portalen der USA erforderlich sein. Ein Zugriff ist theoretisch für alle Ärzte möglich, rechtlich erlaubt aber nur mit Zustimmung des Patienten; zur Kontrolle werden Zugriffsprotokolle geführt. 
Die Planungen zur deutschen elektronischen Gesundheitskarte sehen keine zentrale Sammelstelle für Gesundheitsdaten vor, geplant ist lediglich eine Datenspeicherung auf der Karte sowie eine erleichterte Kommunikation zwischen verschiedenen Gesundheitsanbietern durch einheitliche Schnittstellen. Dennoch ist die Idee eines Datenpools naheliegend und wird oft damit assoziiert. Ein derartiges System zentral verwalteter elektronischer Patientenakten wird derzeit in Großbritannien vorbereitet (http://www.nhscarerecords.nhs.uk/). Bisher war vorgesehen, dass Patienten sich selbst aktiv gegen eine Speicherung bzw. Verwendung ihrer Daten verwehren müssen, wenn sie diese nicht wünschen, seit kurzem ist eine etwas patientenfreundlichere Regelung in Kraft, nach der das Einverständnis der Betroffenen vor Nutzung des Systems eingeholt werden soll.

Die Vorstellung einer derartigen Großdatenbank aus Patientenakten weckt bei vielen Patienten und Ärzten zunächst - und zu Recht - die Angst vor Missbrauch. Eine derartige Datenbank wäre aber auch ein Schatz für die allgemeinmedizinische Forschung, für Qualitätsmanagement und damit auch als Basis einer vernünftigen Planung im Gesundheitswesen. Sie könnte viele Fragen zur Morbidität und Versorgung klären, die in Deutschland bisher unbeantwortet bleiben. Bereits 1999 die Zentrale Ethikkommission in einer Stellungnahme, dass die Verwendung personenbezogener, allerdings anonymisierter Patientendaten für Zwecke der Versorgungsforschung und Qualitätsmanagement auch ohne explizites Einverständnis der jeweiligen Person vertretbar sei, weil nur so Qualitätssicherung möglich wird, die sowohl im gesellschaftlichen als auch individuellen Interesse sei. Bis heute ungeklärt sind allerdings Fragen der Eigentumsrechte an derartigen Daten, ihre Sicherheit sowie Zugriffrechte sind zu klären.
In Zeiten großer persönlicher Mobilität sowie im Sinne des Chronic care Managements und angesichts der Komplexität chronischer oder multipler Erkrankungen kann es auch für den Einzelnen durchaus vorteilhaft sein, wenn Gesundheitsdaten schnell und übersichtlich einsehbar sind; Freiwilligkeit sollte aber Voraussetzung sein.

Vertrauen in die Datensicherheit ist ein zentraler Aspekt, der Ruf zur Vorsicht sehr berechtigt. Ob die Bedenkenträger sich auf die Dauer erfolgreich gegen die informationstechnologischen Entwicklungen im Gesundheitswesen sperren können, darf bezweifelt werden. Parallelschlüsse bieten sich an: Trotz erheblicher, von niemandem bezweifelter Risiken ist die Menschheit nicht bereit, auf das Autofahren zu verzichten. Trotz anfangs erheblicher Sicherheitsbedenken sind Online-Bankgeschäfte ein durchschlagender Erfolg, viele Menschen möchten nicht mehr darauf verzichten. Sie sind schnell, einfach und bequem - und ermöglichen jederzeit guten und schnellen Überblick über den persönlichen Finanzstatus, was einen bewussten Umgang mit Geld erleichtert. Vielleicht werden auch Patienten, die ihre Gesundheitsdaten selbst einsehen und organisieren dürfen, in die Lage versetzt, selbstverantwortlicher handeln zu können.

Was auch immer man davon halten mag - vermutlich ist es nur eine Frage der Zeit, bis sich eine nicht nur in der eigenen Praxis zugängliche digitale Krankenakte auch bei uns durchsetzt. Manchmal siegt eben der Pragmatismus. Als Hausarzt wissen Sie das.

Ihre

Eva Hummers-Pradier und Gudrun Theile als Koautorin 